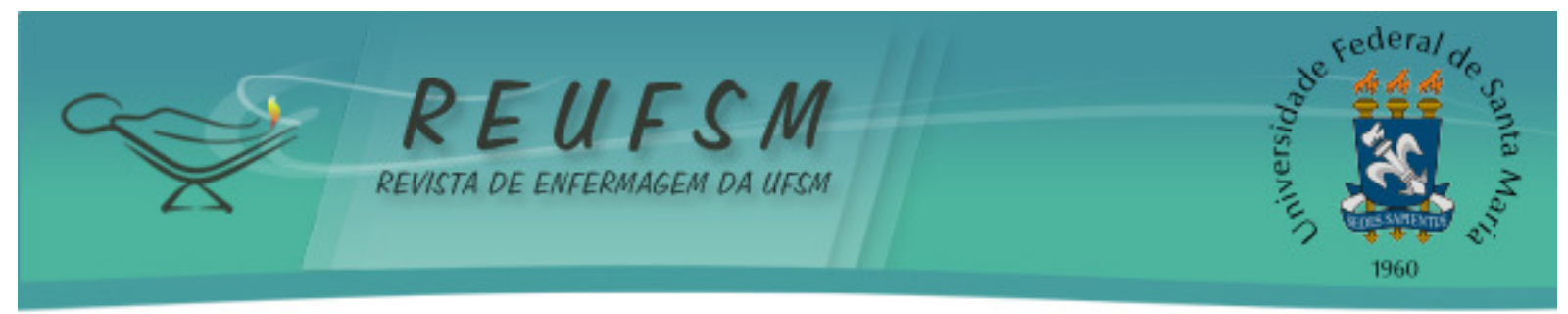

ARTIGO ORIGINAL

\title{
DESAFIOS AO ENFERMEIRO NA IMPLANTAÇÃO DA CLASSIFICAÇÃO DE RISCO EM UNIDADE MISTA
}

\section{CHALLENGES TO THE NURSE IN THEIMPLEMENTATIONOF THE CLASSIFICATION OF RISK IN MIXEDUNIT}

\section{DESAFÍOS AL ENFERMERO EN LA IMPLEMENTACIÓN DE LA CLASIFICACIÓN DE RIESGO EN UNA UNIDAD MIXTA}

Doi: $10.5902 / 2179769210527$

\author{
Cristiano Caveião ${ }^{1}$ \\ Ana Paula $\mathrm{Hey}^{2}$ \\ Juliana Helena Montezeli ${ }^{3}$ \\ Ana Paula de Morais Maia Barros ${ }^{4}$ \\ Janecleia Aparecida Sordi ${ }^{5}$ \\ Sabrina Conde Santos ${ }^{6}$
}

RESUMO: Objetivo: identificar os desafios da implantação do Acolhimento com Classificação de Risco para o enfermeiro classificador em uma unidade mista. Método: trata-se de um estudo descritivo, exploratório de abordagem qualitativa, realizada de agosto a setembro de 2011 por entrevista semiestruturada, com sete enfermeiros de um Centro Municipal de Emergências Médicas do Sul do Brasil, para tratamento dos dados utilizou-se a técnica de Análise de Conteúdo. Resultados: permitiu identificar os seguintes desafios vivenciados pelo enfermeiro classificador: manutenção do fluxo de atendimento correto no Acolhimento com Classificação de Risco; sobrecarga de trabalho e utilização do protocolo classificador. Considerações finais: a sobrecarga de trabalho pode ocasionar a necessidade de um novo dimensionamento de pessoal, pois com mais esta função o enfermeiro poderá se sobrecarregar, podendo gerar afastamentos para tratamento de saúde. A adaptação às queixas dos usuários para inseri-las no protocolo é um desafio para os enfermeiros.

Descritores: Humanização da assistência; Sistemas de saúde; Políticas públicas; Acolhimento.

ABSTRACT: Aim: to identify the challenges of implementation of the Host With Risk Rating for nurses classifier in a mixed unit. Method: this is a descriptive, exploratory qualitative approach, conducted from august to September 2011 by interview semiestrututred, with seven nurses from a Municipal Centre for Medical Emergencies from southern Brazil to treat the data we used the technique of content analysis. Results: identified the following challenges experienced by nurses classifier: maintaining the flow of correct care in Host With Risk Rating; work load and use protocol classifier. Final Thoughts: work overload can cause the need for a new design staff, as this function over the nurse may overload and can cause absenteeism for health treatment. Adapting to user complaints to insert them in the protocol is a challenge for nurses.

Descriptors: Humanization of assistance; Health systems; Public policies; User embracement.

\footnotetext{
${ }^{1}$ Enfermeiro. Doutorando em Enfermagem. Mestre em Biotecnologia pelas Faculdades Pequeno Príncipe (FPP). Professor Pesquisador II das Faculdades Integradas do Brasil - UNIBRASIL. Curitiba - PR, Brasil. E-mail: cristiano_caveiao@hotmail.com

${ }^{2}$ Enfermeira. Mestre pela Pontifícia Universidade Católica do Paraná - PUCPR. Professora da Universidade Tuiuti do Paraná e Faculdade Evangélica do Paraná. Curitiba - PR, Brasil. E-mail: anapaulahey@hotmail.com

${ }^{3}$ Enfermeira. Mestre em Enfermagem pela Universidade Federal do Paraná (UFPR). Professora Assistente no Departamento de Enfermagem da Universidade Estadual de Londrina - UEL. Londrina - PR, Brasil. E-mail: jhmontezeli@hotmail.com

${ }^{4}$ Enfermeira. Prefeitura Municipal de São José dos Pinhais. Curitiba - PR, Brasil. E-mail: anabarros290384@hotmail.com

${ }^{5}$ Enfermeira. Sterilab Curitiba. Curitiba - PR, Brasil. E-mail: jadi_sordi@hotmail.com

${ }^{6}$ Enfermeira. Curitiba - PR, Brasil. E-mail: sassatim@yahoo.com.br
} 


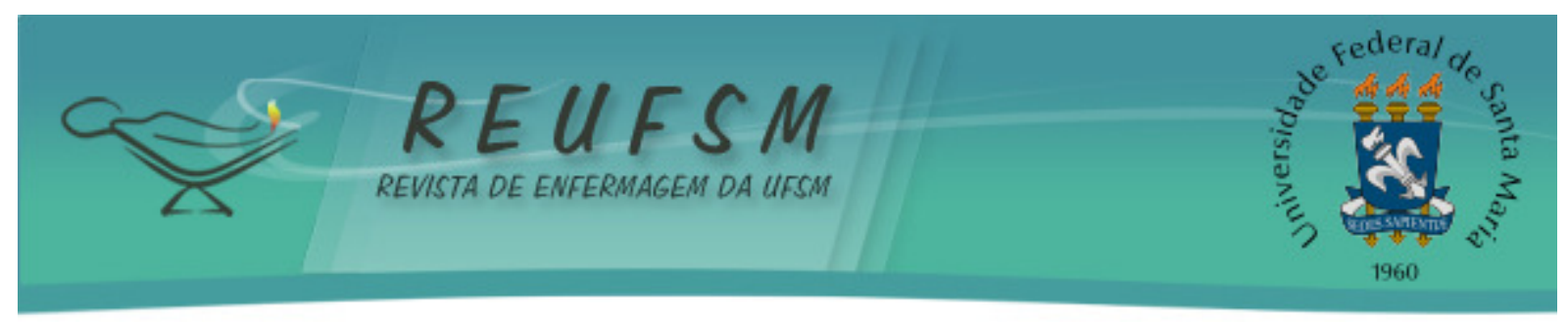

RESUMEN: Objetivo: identificar los desafíos de la implementación del Acogimiento con Clasificación de Riesgo para el enfermero clasificador en una unidad mixta. Método: estudio descriptivo, exploratorio, de abordaje cualitativo realizado de agosto a septiembre de 2011 a través de entrevista semiestrututrada, con siete enfermeros de un Centro Municipal de Emergencias Médicas del Sur de Brasil. Para el tratamiento de los datos se utilizó la técnica de análisis de contenido. Resultados: identificaron los siguientes desafíos experimentados por el enfermero clasificador: manutención del flujo de la atención correcta en el Acogimiento con Clasificación de Riesgo, sobrecarga de trabajo y uso del protocolo clasificador. Consideraciones finales: la sobrecarga de trabajo puede provocar la necesidad de un nuevo dimensionamiento personal, ya que con esta función el enfermero puede sobrecargar, causando ausencia para tratamiento de la salud. Adaptarse a las quejas de los usuarios para insertarlos en el protocolo es un desafío para los enfermeros.

Descriptores: Humanización de la atención; Sistemas de salud; Políticas públicas; Acogimiento.

\section{INTRODUÇÃO}

A busca pelos serviços de emergência tem aumentado progressivamente ao longo dos anos, transformando esta área em um importante componente da assistência à saúde. Muitos destes atendimentos são realizados em unidades mistas, que são conceituadas como unidades de atendimento 24 horas que prestam cuidados de média complexidade e possuem como objetivo estabilizar o paciente para ser encaminhado à unidade especializada ou reinserção na sociedade. Contudo, o aumento da clientela que ali aporta ocorre de maneira desordenada, fazendo com que haja sobrecarga nas mais diferentes portas de entrada emergenciais.

Dentre os fatores que contribuem para essa sobrecarga, podem ser citados o crescimento e envelhecimento populacional, o aumento na incidência dos eventos oriundos de causas externas, doenças cardiovasculares e até a busca de atendimento para problemas simples. ${ }^{1}$

Ao longo deste processo, houve a gênese da necessidade de se organizar o atendimento, porém não de forma excludente expressa em uma simples triagem, mas sim, de maneira acolhedora e considerando a gravidade de cada caso para priorizar a assistência aos mais graves.

Diante deste contexto, criou-se a Política Nacional de Humanização (PNH) vislumbrando a ampliação do acesso da população aos serviços de saúde e maior resolutividade dos problemas com um atendimento acolhedor. ${ }^{2}$

0 protocolo de Manchester ou Acolhimento com Classificação de Risco (ACR) apresenta-se como importante ferramenta para atender a política; foi formulado para utilização no Reino Unido e, atualmente, está em vários países da Europa e Austrália. Com a utilização de protocolos o profissional classificador, mediante treinamentos específicos, ordena a prioridade com base na avaliação do grau de urgência das queixas. ${ }^{3}$ Sendo assim, a classificação não é apenas uma triagem, pois a triagem em seu sentido nato implica numa técnica de escolha de quem será ou não atendido. ${ }^{4}$

Esta proposição do atendimento proporciona a inversão do modelo técnico-assistencial para um modelo usuário-centrado, com maior comprometimento do serviço em relação à defesa individual e coletiva. ${ }^{5}$

Com vistas a atender as preconizações das bases legais sustentadoras desta prática, houve a necessidade dos serviços de emergência adequarem sua organização, tanto do ponto de vista estrutural, quanto de recursos humanos e o enfermeiro insere-se como profissional essencial neste universo.

O modelo proposto pelo Ministério da Saúde sinaliza para que a classificação de risco seja realizada pelo enfermeiro experiente na área de urgência e capacitado para tal. ${ }^{6}$ De acordo com a Resolução $n^{\circ}$. 423/2012 do Conselho Federal de Enfermagem, em seu 


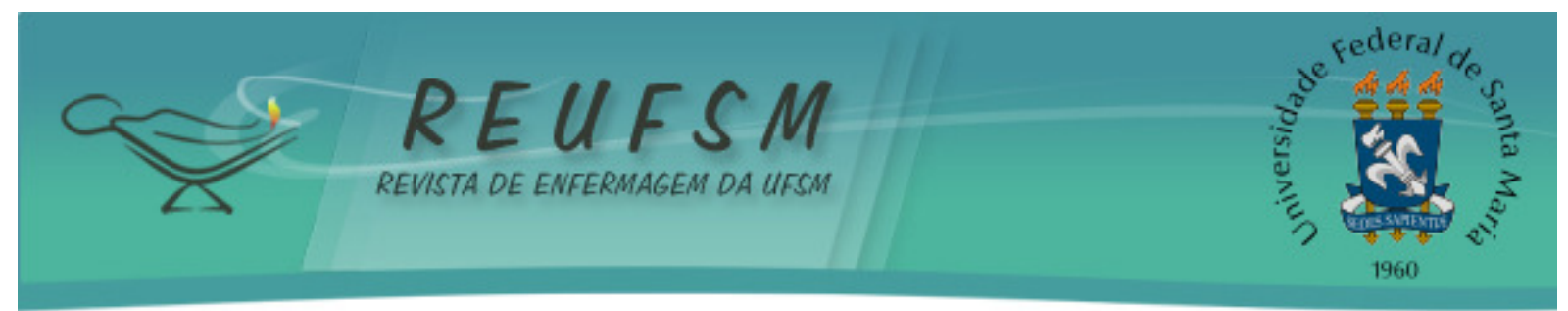

artigo primeiro, no âmbito da equipe de enfermagem, a classificação de risco e priorização da assistência em serviços de urgência é privativa do enfermeiro, observadas as disposições legais da profissão. ${ }^{7}$

Como em todo processo de mudança, os atores envolvidos vivenciam obstáculos que requerem adaptações em seu processo de trabalho. O primeiro passo, então, é conhecer os elementos dificultadores para então traçar estratégias para sua superação.

Destarte, considerando nossa atuação em cenários emergenciais, houve o surgimento de uma série de inquietações que culminaram na realização desta investigação, guiada pela seguinte questão norteadora: quais os desafios da implantação do acolhimento com classificação de risco para o enfermeiro classificador em uma unidade mista? Assim, o objetivo foi: identificar os desafios da implantação do acolhimento com classificação de risco para o enfermeiro classificador em uma unidade mista.

\section{MÉTODO}

Pesquisa descritiva e exploratória de abordagem qualitativa com enfermeiros de um Centro Municipal de Emergências Médicas (CMUM) do Sul do Brasil, durante os meses de agosto e setembro de 2011. A escolha do local ocorreu, pois, a partir do mês de junho do referido ano foi implantado o acolhimento com classificação de risco e os enfermeiros lotados nesta unidade foram capacitados para tal.

Foi realizado com sete enfermeiros de um total de 12 , os quais aceitaram participar do estudo assinando o termo de consentimento livre e esclarecido. Foram excluídos da pesquisa os enfermeiros que estivessem de férias, licença maternidade, licença prêmio ou afastamento por doença e eventuais atestados no período em que foi realizada a coleta de dados, sendo que dois estavam em afastamento por doença, dois não aceitaram participar e um em férias.

O estudo passou por aprovação do Comitê de Ética em Pesquisa da Faculdade do Colégio Brasileiro de Estudos Sistêmicos (CBES) sob o $n^{\circ}$ 255/11, CAEE 0132.0.402.085-11, foram seguidas as recomendações da resolução $466 / 2012^{8}$, que trata de pesquisa com seres humanos. Para a coleta de dados foi utilizado um instrumento estruturado, aplicado pelos pesquisadores composto por uma parte inicial com dados socioprofissionais e por cinco perguntas abertas, relacionadas à Classificação de Risco.

Os dados foram tratados com base na Técnica de Análise de Conteúdo. ${ }^{9}$ Este tipo de análise ocorre em três etapas: a pré-análise (transcrição das falas gravadas dos participantes de forma a constituir o corpus do texto a ser trabalhado), a exploração do material (transformação dos dados brutos em núcleos de compreensão do texto) e o tratamento dos resultados, durante o qual são realizadas inferências e interpretação dos achados, com sustentação de literaturas abordando a temática e exemplificações utilizando fala dos participantes. ${ }^{9}$

\section{RESULTADOS E DISCUSSÃO}

A análise de conteúdo das entrevistas permitiu identificar os seguintes desafios vivenciados pelo enfermeiro classificador: Manutenção do fluxo de atendimento correto no ACR; Sobrecarga de trabalho e Utilização do protocolo classificador. Utilizou-se literatura atual para discussão dos dados. 


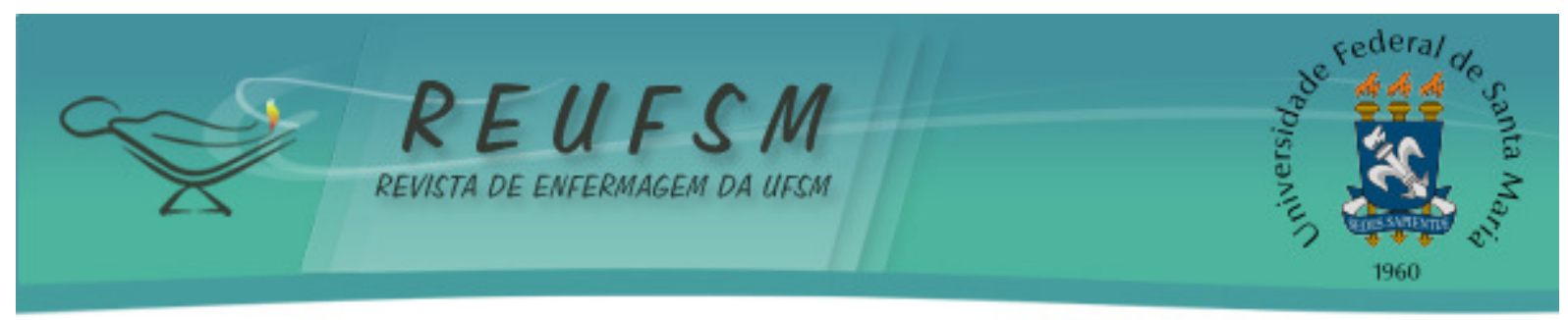

Manutenção do fluxo de atendimento correto no Acolhimento com Classificação de Risco

Evidenciou-se nas falas o perfil da população que busca o atendimento de urgência e emergência. Usualmente trata-se do paciente cujo problema de saúde não é de caráter emergencial, porém, as pessoas entendem este tipo de serviço como meio mais rápido para se conseguir atendimento médico. Isto pode ocorrer pela falta de informação, não entendimento dos níveis de complexidade e credibilidade, fazendo com que ocorra superlotação nestes serviços, como fica evidenciado nos exemplos a seguir:

Em muitos casos o usuário não compreende, por mais que você explique qual é a funcionalidade do CMUM, o que é emergência ou não. Na compreensão deles, esta é uma unidade que deve ficar aberta por 24 horas e atender por 24 horas, independente do motivo da procura. Por isso, muitas vezes a classificação de risco não é entendida. (Violeta)

$O$ atendimento de urgência e emergência do CMUM deveria ser o atendimento e não consulta eletiva. Para isto, as unidades básicas de saúde deveriam estar com o atendimento médico disponível aos usuários eletivos. Quando classificamos pacientes que não são de emergência eles ficam irritados e muitas vezes não aceitam. (Tulipa)

A realidade relatada pelos pesquisados é evidenciada na literatura. Apesar do fluxo hierarquizado, proposto na estruturação do modelo de atenção, este nem sempre é claro para os usuários, os quais acabam por compreender os serviços emergenciais como porta de entrada do sistema de saúde pública. Ao darem acesso aos pacientes que chegam por demanda espontânea e que, em sua maioria, procuram atendimentos de consultas simples e de cuidados básicos, estes cenários acabam por ficar superlotados, com casos que poderiam ser resolvidos no nível primário de atenção. ${ }^{10}$

Entretanto, esta afirmação dos participantes sobre a falta de entendimento dos usuários não é coerente com a PNH e há trabalhos que apontam que não são os usuários que não compreendem, mas que não conseguem acessar outras portas. Exemplifica isso um estudo realizado no hospital universitário do interior do Rio Grande do Sul, o qual permitiu identificar a falta de resolutividade nos níveis básicos da rede de saúde e a falta ou demora no apoio diagnóstico como principais motivos pela busca por atendimento no pronto socorro. Outros motivos elencados pelo referido estudo foram: a necessidade de se chegar muito cedo para acessar uma vaga nos serviços de atenção básica, a demora da consulta em especialidades médicas, a falta de médico na unidade básica e a tecnologia dura disponível no hospital cenário do estudo. ${ }^{11}$

Indubitavelmente esta procura pelos serviços de emergência gera superlotação, a qual fica evidenciada à medida que se analisa a questão da saúde pública no Brasil. Os fatores contribuintes para esse cenário podem ser descritos como a desinformação sobre a regionalização e hierarquização, ineficácia do sistema de referência e contrarreferência das unidades básicas e falta de confiança na resolutividade destes serviços por parte dos usuários. ${ }^{3}$

Assim, o ACR é fundamental para o correto redirecionamento da demanda dos usuários, descongestionando o setor de emergência, e deve ser realizada por profissionais de saúde qualificados para a função, com isso, ocorrerá a resolutividade dos problemas do usuário e melhora no atendimento a saúde da população. ${ }^{10-12}$

Contudo, como a implantação do ACR estava em processo de implantação recente no cenário do estudo, este perfil de clientela que aporta ao serviço representa um dificultador. 


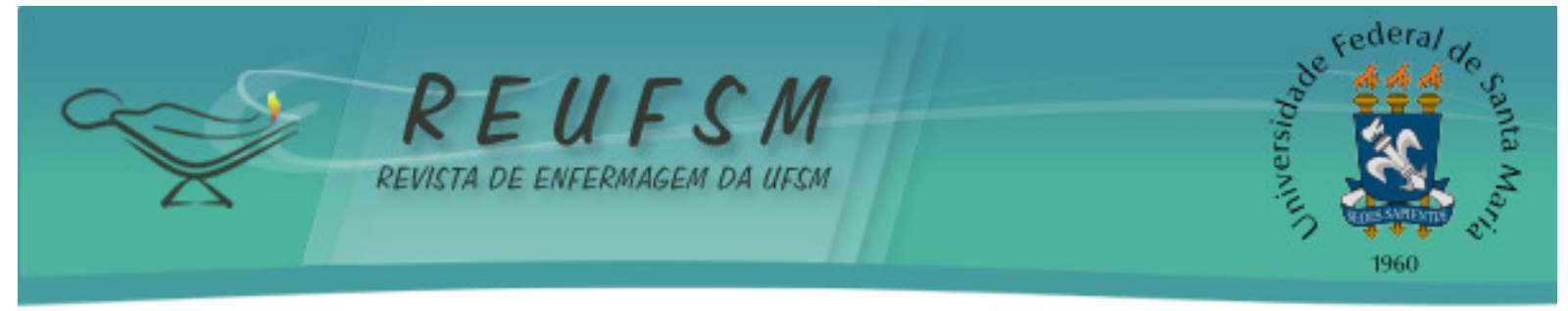

\section{Sobrecarga de trabalho}

Os enfermeiros fizeram referências no tocante à sobrecarga de trabalho oriunda da implantação do ACR, como se percebe nestes trechos:

O estresse gerado em ter que classificar grande número de usuários em pequeno espaço de tempo leva à mecanização do atendimento. Essa dificuldade deve-se ao pouco número de enfermeiros destinados à avaliação de Manchester e a sobrecarga com as demais atividades exigidas. (Hortência)

Sinto-me sobrecarregada, pois além do $A C R$, tenho que resolver simultaneamente problemas de supervisão, realizar procedimento exclusivo de enfermeiro. Nesses momentos, não fica ninguém na avaliação, acumulando usuários para serem classificados. (Rosa)

Com a adoção do sistema de classificação dos pacientes por gravidade clínica, em inúmeros serviços de emergência do Brasil, transferiu-se ao enfermeiro a competência de avaliar o paciente em consonância com protocolos clínicos, que direcionam as necessidades de prioridade e de encaminhamento à área de tratamento. ${ }^{3}$

Sabe-se, porém, que não raramente o enfermeiro classificador assume outras funções concomitantemente, o que pode comprometer a qualidade da avaliação do paciente.

A partir da implantação do ACR surge mais uma função ao enfermeiro e, em contrapartida, cabe refletir sobre a necessidade de reformulação do dimensionamento de pessoal. Acredita-se que a quantidade de enfermeiros dentro das instituições que atendem urgências e emergências deva aumentar, caso contrário, poderá haver sobrecarga de trabalho e queda na qualidade assistencial.

O redimensionamento de enfermeiro é uma questão inevitável para o gerenciamento do cuidado nos serviços de atenção às urgências que dispõem do ACR. Readequar o quantitativo de enfermeiros necessário para o atendimento adequado ao volume de atividades desenvolvidas tem gerado conflitos de natureza econômica, técnica e ética. 0 dimensionamento das equipes e a compatibilização entre a oferta e a demanda por ações de saúde devem ocorrer, conforme preconiza o protocolo de ACR. ${ }^{6}$

\section{Utilização do protocolo classificador}

A principal dificuldade ao executar o ACR, segundo os enfermeiros classificadores, é adaptar as queixas dos usuários e inseri-las no protocolo de maneira a não realizar julgamentos pessoais:

Às vezes o usuário tem dificuldades em expressar seu problema de forma a transformá-lo em uma queixa. Isso dificulta a gente encontrar como classificá-lo de acordo com o protocolo. (Orquídea)

Existem situações em que a queixa não é prevista na classificação. $O$ desafio de seguir o protocolo a partir das queixas do paciente e não em julgamento particular do classificador. (Margarida)

A classificação de risco é um processo dinâmico de identificação de pacientes que necessitam de tratamento imediato. Isto é desenvolvido de acordo com o potencial de 


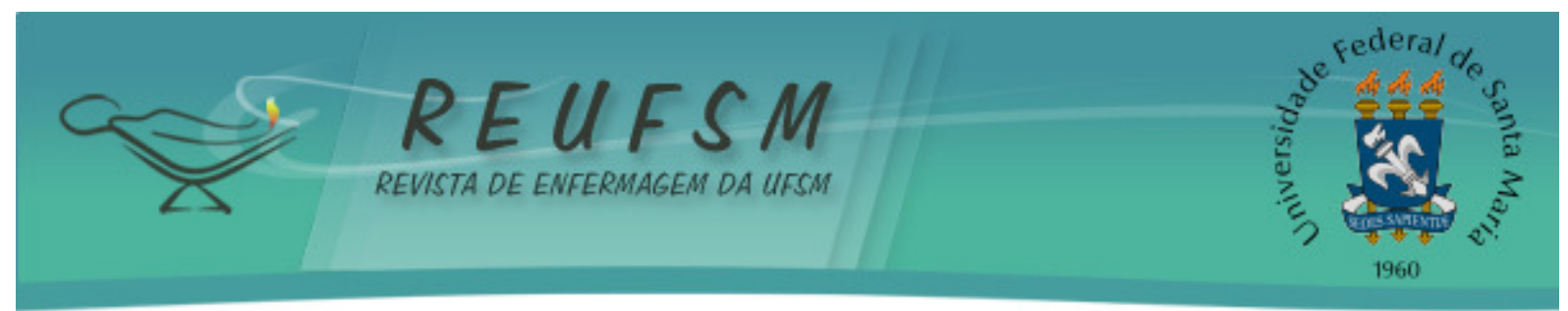

risco, agravo à saúde, ou grau de sofrimento, devendo o atendimento ser priorizado conforme a gravidade clínica do paciente e não com a ordem de chegada ao serviço. ${ }^{13}$

Corroborando com as falas, o processo de adaptação da queixa do paciente ao fluxograma do protocolo é um dos processos mais difíceis, pois exige deste profissional escuta qualificada, avaliação e registro correto e detalhado da queixa principal, capacidade de observação, raciocínio clínico, tomada de decisão e conhecimento das redes de apoio do sistema assistencial. ${ }^{4}$

Ainda, o fluxograma no qual a queixa do paciente é direcionada e sequencialmente classificada, muitas vezes não se correlaciona com o relato do paciente. Isto requer uma habilidade de entendimento e interpretação maior por parte do profissional que realiza a classificação, sendo assim é fundamental a análise do enfermeiro, tendo uma visão clínica e mais profunda da queixa do usuário. ${ }^{4}$

Desta maneira, é fundamental que o enfermeiro classificador reconheça a educação permanente como aliada ao longo da vida profissional, com vistas a adquirir e manter um lastro de conhecimento que possa embasar seu raciocínio clínico no processo de classificação.

A educação permanente quando estimulada visa a valorização dos enfermeiros que atuam com ACR, pois proporciona maior integração entre os trabalhadores e os usuários do sistema de saúde. Envolvem a articulação entre educação e trabalho no SUS, visando à produção de mudanças nas práticas de formação e de saúde, pois permite o desenvolvimento da capacidade pedagógica de problematizar e identificar pontos sensíveis e estratégicos para a produção da integralidade e humanização. ${ }^{2,14}$

\section{CONSIDERAÇÕES FINAIS}

Destacam-se as contribuições que este estudo trouxe acerca dos desafios enfrentados pelos enfermeiros na implantação do ACR neste cenário. Possibilitaram-se algumas reflexões em relação às necessidades de políticas públicas de saúde mais participantes, em que a conscientização da população é fundamental, pois muitas pessoas ainda entendem este tipo de serviço como meio mais rápido para se conseguir atendimento médico.

Corroborando, há a necessidade de uma divulgação mais intensa, no que se refere às mudanças do modelo de atendimento, para que o usuário tenha conhecimento sobre o que é uma situação de emergência e uma situação eletiva, e qual serviço ele deverá buscar para ter o atendimento adequado. Em contrapartida, é essencial que haja resolutividade do problema do usuário, necessitando que o mesmo seja acolhido no sistema de saúde. Para tal, é preciso que os diferentes níveis de atenção sejam eficazes e eficientes nas suas respectivas atribuiçõos.

Em relação à sobrecarga de trabalho, ressalta-se a necessidade de um novo dimensionamento de pessoal, pois com mais esta função o enfermeiro poderá ter a sua saúde prejudicada, além de influenciar na qualidade de suas ações.

Distante de sanar as discussões sobre a temática, enfatiza-se que a PNH prevê a flexibilização da hierarquia do SUS, sendo que o ACR deve ser um método que se distancia da triagem excludente nas portas de entrada. Porém, a efetivação desta mudança ainda apresenta diversas limitações, indo aquém dos limites e possibilidades da equipe, e dependendo muitas vezes de vontade política e das mudanças estruturais dos serviços de saúde.

Sendo assim, vislumbra-se que os resultados da presente investigação, mesmo com suas limitações, possam servir como fulcro para outros estudos que não apenas identifiquem os obstáculos para a prática do ACR, mas também possibilitem intervenções para melhoria nos cenários emergenciais e, consequentemente, na assistência à saúde da população.

Cabe, então, a população lutar pelos seus direitos, ao Estado informar e melhorar as políticas de saúde, fiscalizando sua implementação e fornecendo condições para tal, e, 


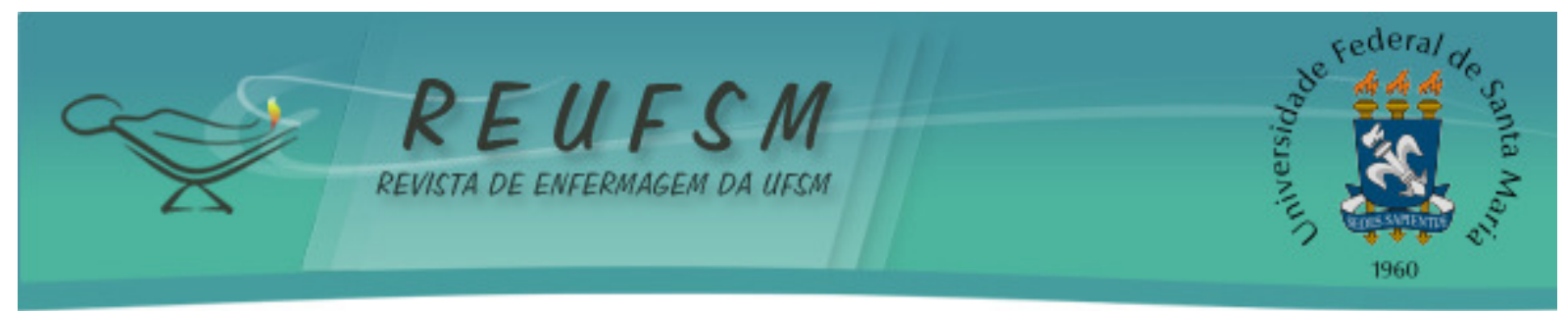

por fim, aos profissionais a busca permanente de conhecimentos para a realização do ACR com responsabilidade, empatia, respeito ao próximo e verdadeiramente humanizado.

\section{REFERÊNCIAS}

1. Madeira DB, Loureiro GM, Nora EA. Classificação de risco: perfil do atendimento em um Hospital Municipal do Leste de Minas Gerais. Rev Enferm Int [Internet]. 2010 nov/dez [acesso em 2013 ago 10];3(2):543-53. Disponível em: http://www.unilestemg.br/enfermagemintegrada/artigo/V3_2/07-classificacao-de-riscoperfil-atendimento-hospital-municipal.pdf.

2. Brasil. Ministério da Saúde. Secretaria Executiva. Núcleo Técnico da Política Nacional de Humanização. HumanizaSUS: política nacional de humanização: documento base para gestores e trabalhadores do SUS. Brasília (DF): Ministério da Saúde; 2004.

3. Ulhôa ML, Garcia FC, Lima CT, Santos DS, Castro PAA. A implantação de nova tecnologia: implicação na eficiência do trabalho na unidade de pronto atendimento de um hospital público de urgência e emergência. Rev Gest Organ [Internet]. 2010 dez [acesso em 2013 ago 10];3(1):99-118. Disponível em: http: //www.spell.org.br/documentos/download/1814.

4. Souza CC. Grau de concordância da classificação de risco de usuários atendidos em um pronto socorro utilizando dois diferentes protocolos [dissertação].Belo Horizonte (MG): Universidade Federal de Minas Gerais; 2009 [acesso em 2013 ago 10]. Disponível em: http: //www.bibliotecadigital.ufmg.br/dspace/handle/1843/GCPA-83FKCZ.

5. Zem KKS, Montezeli JH, Peres AM. Acolhimento com classificação de risco: concepção de enfermeiros de um pronto socorro. Rev RENE [Internet]. 2012 abr [acesso em 2013 ago 10];13(4):899-908. Disponível

em: http://www.revistarene.ufc.br/revista/index.php/revista/article/view/1086.

6. Brasil. Ministério da Saúde. Secretaria de atenção à saúde. Política nacional de Humanização da atenção e Gestão do SUS. Acolhimento e classificação de risco nos serviços de urgência. Brasília (DF): Ministério da Saúde; 2009. (série B. textos Básicos de saúde).

7. Conselho Federal de Enfermagem. Resolução COFEN n 423, de 9 de abril de 2012. Normatiza, no âmbito do sistema Cofen/Conselhos Regionais de Enfermagem, a participação do enfermeiro na atividade de classificação de riscos. Brasília (DF): COFEN; 2012.

8. Ministério da Saúde (BR). Conselho Nacional de Saúde. Resolução n. 466, de 12 de dezembro de 2012. Aprova diretrizes e normas regulamentadoras de pesquisas envolvendo seres humanos e revoga também as Resoluções no 196/1996, n- 303/2000 e no 404/2008. Brasília (DF): Ministério da Saúde; 2012. Disponível em: http://conselho.saude.gov.br/resolucoes/2012/Reso466.pdf.

9. Bardin L. Análise de conteúdo. Lisboa: Edições 70; 2011.

10. Morishita A, Silva EA, Souza MAM. Concepção de triagem X demanda do atendimento em unidades de urgência e emergência. Rev Ponto Encon. 2009;1:196-209.

11. Casarotto M, Schimith MD, Budó MLD, Weiller TH, Simon BS. "Eu fui ao posto mais próximo...": livre demanda do pronto socorro de um hospital universitário. Rev Pesq Cuid Fundam [Internet]. 2012 jul/set [acesso em 2013 nov 10];4(3):2712-21. Disponível em: http://www.seer.unirio.br/index.php/cuidadofundamental/article/view/1547/pdf_611. 


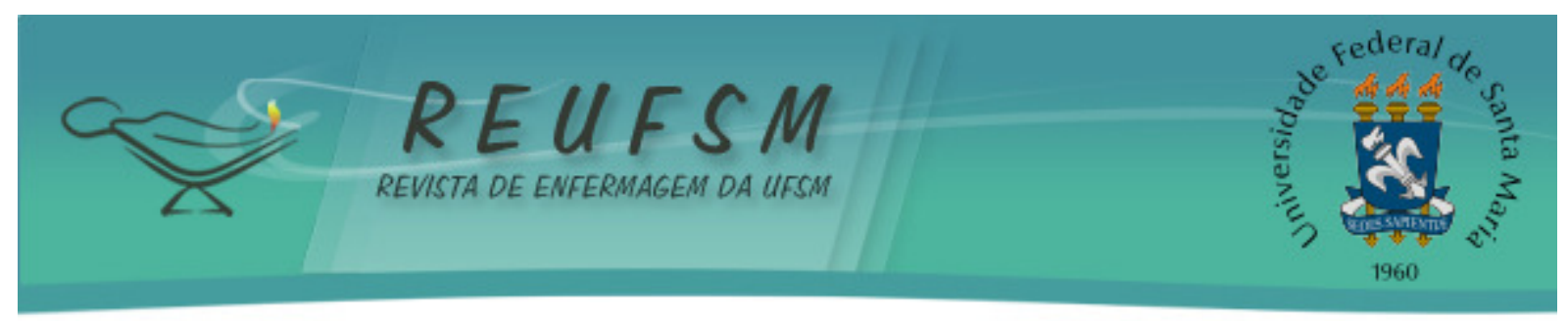

12. Shiroma LMB, Pires DEP. Classificação de risco em emergência - um desafio para as/os enfermeiras/os. Enferm Foco [Internet]. 2011 jan [acesso em 2013 ago 10];2(1):14-7. Disponível em: http://revista.portalcofen.gov.br/index.php/enfermagem/article/view/67/54.

13. Souza CC, Toledo AD, Tadeu LFR, Chianca TCM. Classificação de risco em prontosocorro: concordância entre o protocolo institucional brasileiro e Manchester. Rev Latinoam Enferm [Internet]. 2011 jan-fev [acesso em 2013 ago 10];19(1):(8 telas). Disponível em: http://www.scielo.br/pdf/rlae/v19n1/pt_05.pdf.

14. Silva LAA, Bonacina DM, Andrade A, Oliveira TC. Desafios na construção de um projeto de educação permanente em saúde. Rev Enferm UFSM [Internet]. 2012 set/dez [acesso em 2013 ago 10];2(3):496-506. Disponível em: http://cascavel.ufsm.br/revistas/ojs2.2.2/index.php/reufsm/article/view/5364/pdf.

Data de recebimento: 29/08/2013

Data de aceite: 02/12/2013

Contato com autor responsável: Cristiano Caveião

Endereço postal: Rua Osório dos Santos Pacheco, 675

E-mail: cristiano_caveiao@hotmail.com 